\title{
Regulation of Gdnf expression by retinoic acid in Sertoli cells
}

\author{
Rossana Saracino | Chiara Capponi | Sara Di Persio | Carla Boitani | \\ Silvia Masciarelli | Francesco Fazi | Stefania Fera | Elena Vicini
}

Department of Anatomy, Histology, Forensic Medicine and Orthopedic, Section of Histology, Sapienza University of Rome, Roma, Italy

\section{Correspondence}

Elena Vicini, Department of Anatomy, Histology, Forensic Medicine and Orthopedic, Section of Histology, Sapienza University of Rome, Via Antonio Scarpa 16, 00161 Roma, Italy.

Email: elena.vicini@uniroma1.it

Funding information

Sapienza Università di Roma,

Grant/Award Number: Ateneo 2018

\begin{abstract}
Glial cell line-derived neurotrophic factor (GDNF) and retinoic acid (RA) are two molecules crucial for the regulation of the spermatogonial compartment of the testis. During the cycle of the seminiferous epithelium, their relative concentration oscillates with lower GDNF levels in stages where RA levels are high. It has been recently shown that RA negatively regulates $G d n f$ expression but the mechanisms behind are so far unknown. Here, we show that RA directly downregulates Gdnf mRNA levels in primary murine Sertoli cells through binding of RAR $\alpha$ to a novel DR5-RARE on Gdnf promoter. Pharmacological inhibition and chromatin immunoprecipitation-quantitative polymerase chain reaction analysis suggested that the underlying mechanism involved histone deacetylase activity and epigenetic repression of Gdnf promoter upon RA treatment.
\end{abstract}

\section{KEYWORDS}

cycle of the seminiferous epithelium, GDNF, retinoic acid, Sertoli cells

\section{1 | INTRODUCTION}

The continuous production of mature sperm in adult mammalian testis is sustained by the biological activity of spermatogonia. They are located at the basal region of the seminiferous tubules, in close contact with the Sertoli cells and the basement membrane. The spermatogonial compartment comprises the spermatogonial stem cells (SSC), the undifferentiated and the differentiating spermatogonia. The factors implicated in the homeostatic control of this compartment include two crucial molecules, the glial cell line-derived neurotrophic factor (GDNF) and retinoic acid (RA) (Boitani, Di Persio, Esposito, \& Vicini, 2016). GDNF is a major paracrine factor regulating the self-renewal of SSC both in vivo and in vitro (Kanatsu-Shinohara et al., 2003; Meng et al., 2000). In mice with Gdnf haploinsufficiency, there is a depletion of the SSC pool (Meng et al., 2000). In contrast, when Gdnf is ectopically expressed, QJ;large clusters of single type A spermatogonia appear inside the seminiferous tubules and ultimately seminomatous tumors are formed (Meng, de Rooij, Westerdahl, Saarma, \& Sariola, 2001; Meng et al., 2000; Sharma \& Braun, 2018; Yomogida, Yagura, Tadokoro, \& Nishimune, 2003). Recently, it was proposed that, rather than stimulating the proliferation of SSC, the GDNF impacts on SSC by blocking their differentiation (Sharma \& Braun, 2018). Interestingly, GDNF can function in vitro as a chemoattractant for undifferentiated spermatogonia (Dovere et al., 2013). Moreover, GDNF-soaked beads transplanted in mouse testis induce a rapid local aggregation of GFRA1-expressing spermatogonia around the beads (Uchida et al., 2016). Taken together, these data suggest that GDNF attracts or maintains SSC in their niche thereby inhibiting their differentiation. The observation that GFRA1, the coreceptor for GDNF, is expressed in most of undifferentiated spermatogonia, suggests that the role of GDNF may not be restricted to SSC (Grasso et al., 2012).

$\mathrm{RA}$, the active metabolite of vitamin A, regulates spermatogonial differentiation (Griswold, 2016). In vitamin A-deficient (VAD) mice and rats, most germ cells arrest as undifferentiated spermatogonia (van Pelt \& de Rooij, 1990a, 1990b). When VAD animals are injected with RA or vitamin A, the arrested spermatogonia differentiate (Morales \& Griswold, 1987; van Pelt \& de Rooij, 1991). The different enzymatic components required for the synthesis, signaling, and degradation of RA are expressed in the testis. Furthermore, both germ and Sertoli cells in the seminiferous epithelium can produce, use, and degrade RA. As a result, the local concentration of RA is finely tuned (Griswold, 2016). 
Within target cells, RA interacts with heterodimers of the RA receptors (RARs) and the retinoid $X$ receptor ( $R X R$ ), which in turn, binds RA response elements (RAREs) on the regulatory regions of RA-responsive genes. These RAREs are composed of two hexameric $\mathrm{DR}$ (direct repeats) spaced by 5 bp (DR5) or 2 bp (DR2). For both DR5 and DR2, the $5^{\prime}$ of the sequence is bound by RXR and the 3 ' by RAR (Cunningham \& Duester, 2015). In the absence of RA, the RAR/RXR heterodimer is bound to the RARE sequence and inhibits the transcription of the target genes, by recruiting corepressors, histone deacetylases (HDACs) and the repressive chromatin-remodeling complex. This leads to increased histone methylation, chromatin condensation, and gene silencing. When RA binds to the RAR/RXR heterodimer, it promotes the release of repressors, the recruitment of coactivators and histone acetyltransferase (HATs), the erasure of negative epigenetic modifications and the induction of activating transcription modifications. These modifications reverse chromatin condensation, allowing the transcription factors and the RNA polymerase to access the promoter. However, in contrast to the classical model, there are a few cases where the binding of RA to the RAR/ RXR leads to repression of transcription through the recruitment of the Polycomb complex and the accumulation of repressive changes. Some examples of RA negatively-regulated genes are fibroblast growth factor (Fgf8), homeobox gene B1 (Hoxb1), Pou5f3, and Wnt8a (Cunningham \& Duester, 2015).

In the seminiferous tubules, local levels of both GDNF and RA oscillate during the cycle of the seminiferous epithelium (Endo et al., 2015; Grasso et al., 2012; Hasegawa, Namekawa, \& Saga, 2013; Hogarth et al., 2015; Johnston, Olivas, DiCandeloro, \& Wright, 2011; Sharma \& Braun, 2018). Thus, GDNF levels are lower when the concentration of RA is high, suggesting a negative regulation of Gdnf by RA. Evidence of RA-mediated Gdnf inhibition was first reported by Hasegawa et al. (2013). The administration of RA in both cultured Sertoli cells and VAD mice induced a reduction in Gdnf expression, while overexpression of a dominant-negative RAR $\alpha$ in Sertoli cells induced the upregulation of Gdnf during stages VII-XII, when RA levels are typically high (Hasegawa et al., 2013). More recently, it was shown that systemic administration of RA in mice reduces the expression of Gdnf at testicular level (Sakai, Masaki, Aiba, Tone, \& Takashima, 2018). However, the mechanisms underlying the negative regulation of RA on Gdnf expression levels are unclear. In this study we examined the molecular mechanisms by which RA negatively affects the expression of Gdnf in Sertoli cells.

\section{RESULTS}

\subsection{RA treatment inhibits Gdnf mRNA levels in ex vivo cultured testis}

We first analyzed whether the negative regulation of Gdnf mRNA levels by RA was reproduced in ex vivo cultured testis. To this end, testis organ cultures were prepared from immature and adult mice and treated for 8 hr in the presence or absence of 1 or $10 \mu \mathrm{M}$ RA. In immature testis, Gdnf was downregulated at both RA concentrations compared with untreated samples (Figure 1a). As internal control of treatment efficacy, the induction of Stra6 transcript, a well-known RA-responsive gene, was evaluated (Bouillet et al., 1997). As expected, in immature testis, Stra6 was induced at both RA concentrations (Figure S1a). On the contrary, in adult testis, neither of the two RA concentrations lowered Gdnf mRNA levels (Figure $1 \mathrm{~b})$. In adult testis, RA $(1 \mu \mathrm{M})$ was able to increase Stra6 mRNA, although not significantly (Figure S1b).

Because in adult testis the concomitant presence of multiple germ cell associations could hide a stage-specific gene regulation, we analyzed the impact of RA on specific stages of the seminiferous epithelium. By using transillumination microscopy, we isolated stages I-VI, VII-VIII, and IX-XI seminiferous tubule fragments, and cultured them in vitro in the presence or absence of RA. In line with our previous results, the Gdnf levels were high in stages II-VI and lower in stages VII-VIII and IX-XI (Figure 1c; Grasso et al., 2012; Lamberti \& Vicini, 2014). The treatment with RA significantly inhibited Gdnf levels in stages II-VI but not in stages VII-VIII and IX-XI (Figure 1c). Stra6 mRNA basal levels were also stage-dependent, very low at stages II-VI and IX-XI and high at stages VII-VIII. Similarly, RA-stimulation induced Stra6 expression only in stages II-VI (Figure S1c). These data suggest that the RA-mediated regulation of Gdnf mRNA is reproduced in ex vivo cultured testis.

\subsection{RA inhibits Gdnf mRNA levels in primary Sertoli cells}

In the murine testis Gdnf is expressed by Sertoli cells (Golden, DeMaro, Osborne, Milbrandt, \& Johnson, 1999; Tadokoro, Yomogida, Ohta, Tohda, \& Nishimune, 2002), peritubular cells (Chen, Willis, \& Eddy, 2016), and endothelial cells (Bhang et al., 2018). To deepen the mechanism of RA-mediated Gdnf inhibition, we used primary Sertoli cell cultures, prepared from immature mice. Sertoli cells were treated in the presence of increasing concentrations of RA (0.01-10 $\mu \mathrm{M}$; Figure 2a). The results showed a dose-dependent negative modulation of Gdnf with a significant effect at $1 \mu \mathrm{M}$ RA. As expected, Stra6 was induced at all RA concentrations (Figure S2). Time-course analysis, of RA-dependent Gdnf regulation, showed a significant reduction of Gdnf mRNA ( 40\%) after $2 \mathrm{hr}$ of treatment that was maintained up to $24 \mathrm{hr}$ (Figure 2b). To evaluate whether the inhibition of Gdnf was driven by regulation of transcriptional stability or protein synthesis, Sertoli cells were treated for $2 \mathrm{hr}$ in the presence of actinomycin D (ActD) or cycloheximide $(\mathrm{CHX})$, with or without RA. Interestingly, act D treatment alone induced a $80 \%$ reduction of Gdnf mRNA levels compared with the control, indicating a short half-life of Gdnf mRNA (Figure 2c). RA and ActD cotreatment was similar to ActD alone. In contrast, treatment with $\mathrm{CHX}$ increased Gdnf mRNA levels, suggesting that an ongoing synthesis of a transcriptional repressor controls the basal Gdnf levels. However, in cells treated with $\mathrm{CHX}$, RA inhibited mRNA levels compared with control, suggesting that the mechanism of action of RA on Gdnf transcript does not require protein neosynthesis. These data indicate that in Sertoli cells Gdnf expression levels are directly inhibited by RA. 
(a)

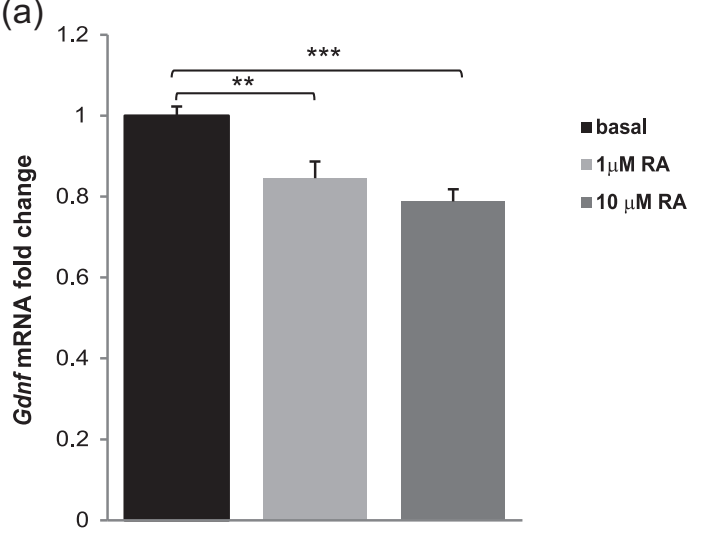

(b)

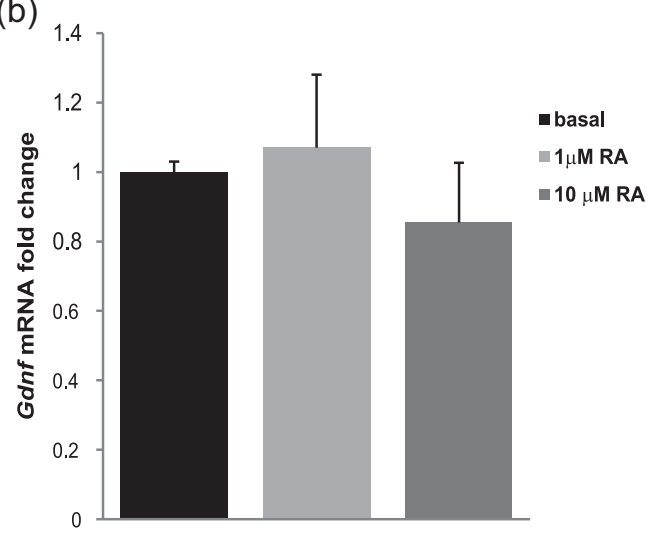

(c)

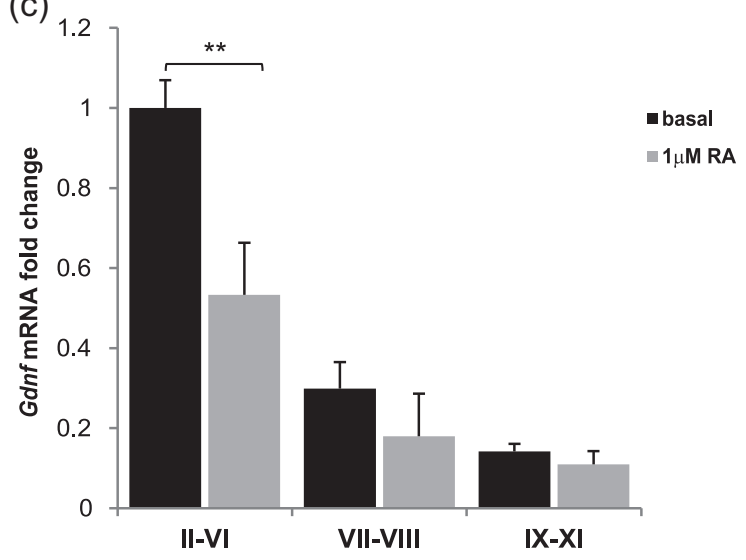

FIGURE 1 RA treatment inhibits Gdnf mRNA levels in ex vivo cultured testis. (a) Immature, and adult (b) murine testicular fragments were cultured for $8 \mathrm{hr}$ treatment in the presence or absence of 1 or $10 \mu \mathrm{M}$ RA. (c) Staged seminiferous tubules (II-VI, VII-VIII, IX-XI) were isolated and cultured for $4 \mathrm{hr}$ in the presence or absence of $1 \mu \mathrm{M}$ RA. Gdnf mRNA levels were measured by real-time PCR, normalized to $\beta$-actin levels and expressed as fold change with respect to basal conditions in ( $a$ and b), while in (c) the reference point is the basal condition of stages II-VI. Data are shown as mean \pm SEM from $n=3$ experiments. ${ }^{* *} p<.01,{ }^{* * *} p<.001$ versus basal conditions with Student-Newman-Keuls Method. mRNA, messenger RNA; RA, retinoic acid; PCR, polymerase chain reaction; SEM, standard error of the mean

\section{3 | Identification of a novel RARE-DR5 on the Gdnf promoter region}

We next asked whether the action of RA on Gdnf mRNA levels involved the $5^{\prime}$-flanking regulatory region of the Gdnf gene. Sertoli cells were transiently transfected with the 5 '-flanking region of Gdnf cloned upstream of the luciferase reporter gene $(-1,857 /+109-p G L 2)$ and treated in the presence or absence of $1 \mu \mathrm{M}$ RA for $24 \mathrm{hr}$ (Lamberti \& Vicini, 2014). In transfected Sertoli cells, RA treatment reduced the luciferase activity by $40 \%$ compared with basal condition, recapitulating the effect of RA on the endogenous level of Gdnf transcripts (Figure 3a). These results indicate that the 5 '-flanking region contains a genomic sequence implicated in the negative regulation of mRNA levels exerted by RA. We then performed an in silico analysis to detect putative RARE on the murine Gdnf promoter. A putative RARE-DR5 sequence (TGACCTCTCCCTGATCA) was identified in reverse orientation with respect to Gdnf gene, just upstream of the three CRE regions (Lamberti \& Vicini, 2014; Figure 3b). Genomic multiple alignment showed that, while the putative mouse
RARE-DR5 has two base mismatches compared with the canonical RARE-DR5, rat and human showed a minor degree of similarity to the canonical sequence (Figure S3; Balmer \& Blomhoff, 2005). To analyze the involvement of the putative RARE-DR5 in RA-mediated regulation, this region was deleted in $-1,857 /+109$ genomic fragment $(-1,857 /+109-\Delta$ (RARE-DR5)-pGL2), and the luciferase activity measured in Sertoli cells transfected in the presence or absence of RA. The internal deletion of the putative RARE-DR5 binding site reversed the inhibition of RA treatment on luciferase activity (Figure 3a). This suggests that the RARE-DR5 sequence identified in silico is involved in RA-mediated regulation.

\section{4 | RAR $\alpha$ is implicated in the RA-mediated Gdnf regulation}

To determine whether the downregulation of Gdnf is due to RARs, Sertoli cells were treated with BMS-204493, an inverse agonist inhibitor of all three RARs isoforms (RAR $\alpha, R A R \beta$, and RAR $\gamma$; 


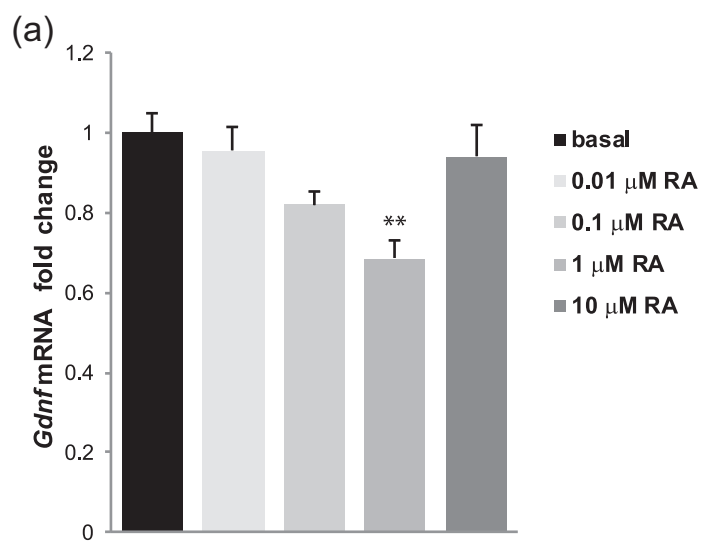

(c) (b)

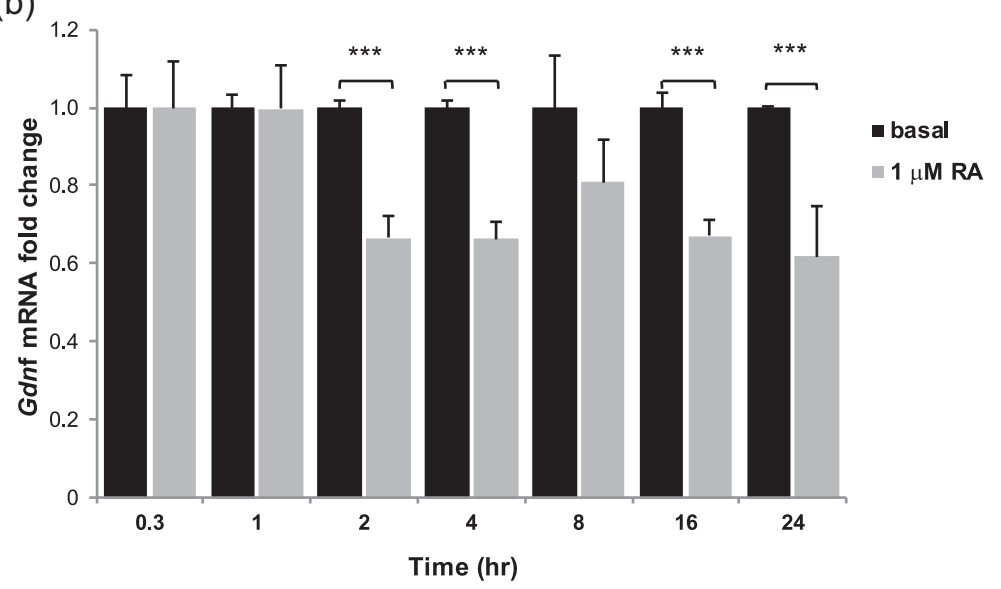

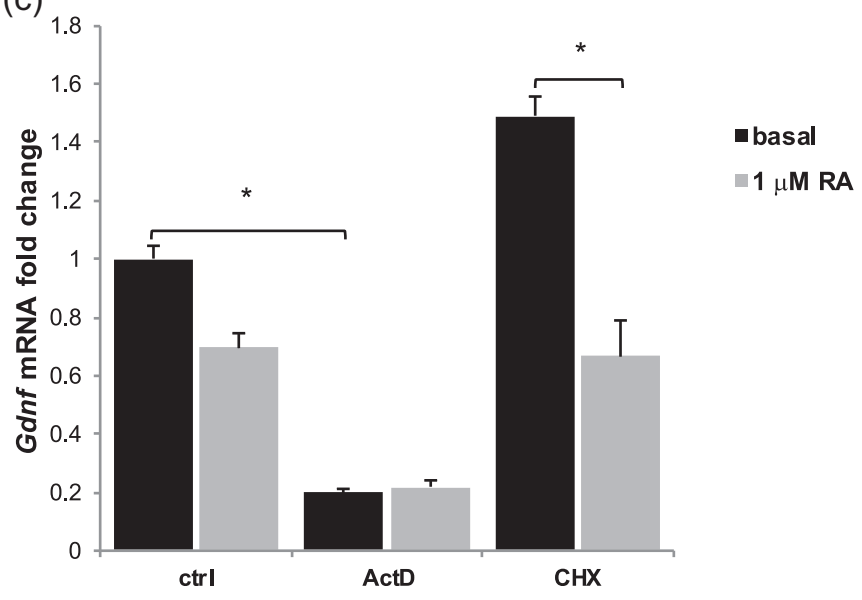

FIGURE 2 RA inhibits Gdnf mRNA levels in a dose- and time-dependent fashion. (a and b) Sertoli cells were cultured for 24 hr with or without increasing concentrations of RA (a), or for different time lengths in the presence or absence of $1 \mu \mathrm{M}$ RA (b). Gdnf mRNA levels were measured by real-time PCR, normalized to $\beta$-actin levels and expressed as fold change with respect to basal conditions. Data are shown as mean \pm SEM from $n=3$ experiments. ${ }^{* *} p<.01,{ }^{* * *} p<.01$ versus basal conditions with Student-Newman-Keuls Method. (c) Sertoli cells were cultured for $2 \mathrm{hr}$ in control conditions or with $5 \mu \mathrm{g} / \mathrm{ml} \mathrm{ActD}$, or with $10 \mu \mathrm{g} / \mathrm{ml} \mathrm{CHX}$ in the presence or absence of RA. Gdnf mRNA levels were quantified by real-time PCR, normalized to $\beta$-actin levels and expressed as fold change respect to untreated control. Data are expressed as mean $\pm S E M$ from $n=2$ experiments and expressed in fold change compared with the untreated control. ${ }^{*} p<.05$. ActD, actinomycin $D$; CHX, cycloheximide; mRNA, messenger RNA; RA, retinoic acid; PCR, polymerase chain reaction; SEM, standard error of the mean

Raverdeau et al., 2012). Treatment with BMS-204493 alone did not modulate Gdnf baseline levels but it reversed the RA-mediated downregulation (Figure 4a). Since RAR $\alpha$ is the predominant RAR in Sertoli cells (Hasegawa \& Saga, 2012; Vernet et al., 2006), we investigated the involvement of this isoform using RO41-5253, a selective antagonist of RAR $\alpha$ (Ertesvag, Aasheim, Naderi, \& Blomhoff, 2007). In the presence of RO41-5253, the inhibitory effect of RA on Gdnf transcript was reversed, while the basal levels were not affected (Figure 4b). To further show the involvement of RAR $\alpha$, we next examined the effect of its ablation by small RNA interference (siRNA). In Sertoli cells, RAR $\alpha$ siRNA lowered RAR $\alpha$ mRNA levels by around 70\% compared with the scramble sequence (Figure S4). As expected, in Sertoli cells transfected with a scramble RNA, RA was able to downregulate Gdnf mRNA. Conversely, in cells where RAR $\alpha$ was interfered, RA did not lower Gdnf levels (Figure 4c). This approach confirmed the RAR $\alpha$ involvement in Gdnf regulation and strengthened previous conclusions obtained using the pharmacological inhibitors. Finally, to directly demonstrate RAR $\alpha$ occupancy on the putative RARE-DR5, we performed chromatin immunoprecipitation-quantitative polymerase chain reaction (ChIP-qPCR) experiments. Sertoli cells were cultured with or without RA for $2 \mathrm{hr}$, and the enrichment mediated by the RAR $\alpha$ antibody was measured (Figure $4 \mathrm{~d}$ ). The results demonstrate that RAR $\alpha$ occupies the RARE-DR5 both under basal condition and in RA-treated cells. Altogether, these data indicated that RAR $\alpha$ mediates the effect of RA through the RARE-DR5.

\section{5 | Epigenetic mechanisms regulate the RA-mediated inhibition of Gdnf}

In the few documented cases of direct genomic repressive action mediated by RA, the recruitment of HDACs seems to be involved 
(a)

(b)

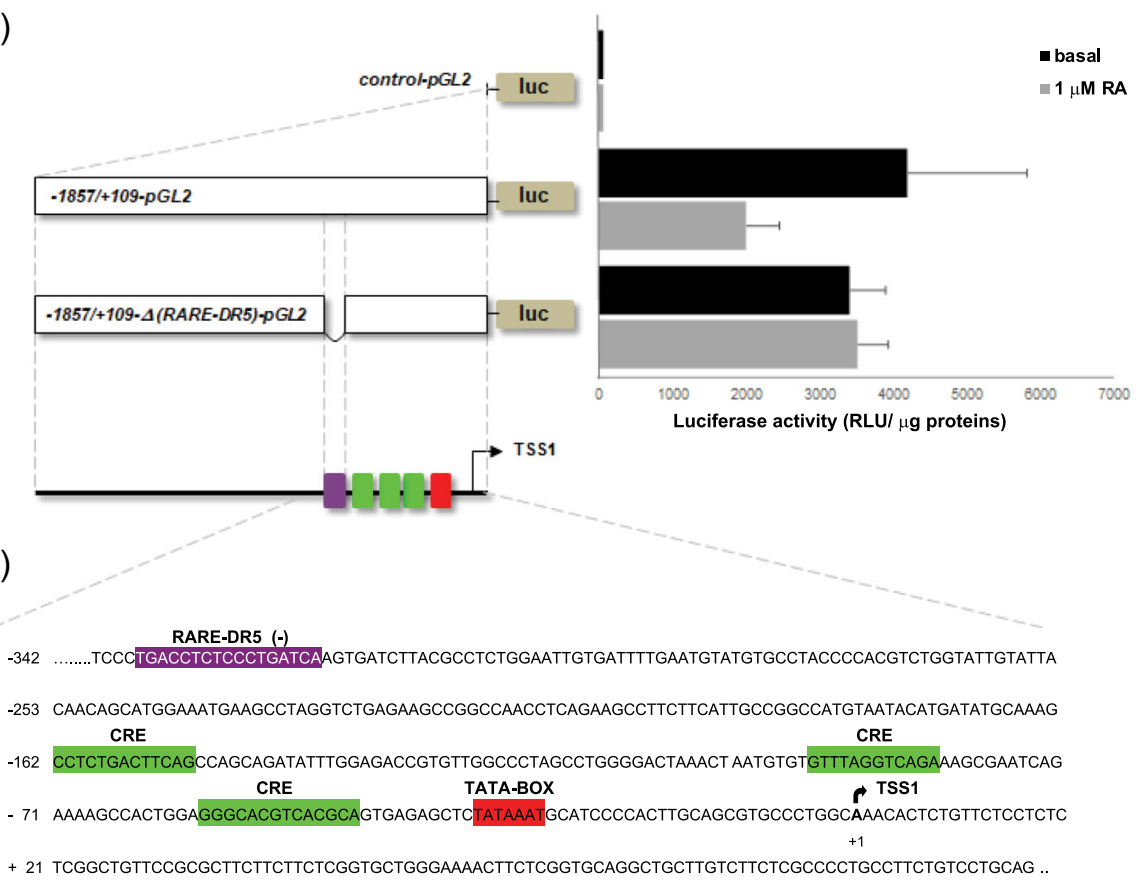

FIGURE 3 Involvement of novel identified RARE-DR5 element on the RA-mediated Gdnf inhibition. (a) Sertoli cell cultures were transfected with indicated plasmids and treated with vehicle (basal conditions) or with $1 \mu \mathrm{M}$ RA for 24 hr. Luciferase activity is expressed as RLU, corrected by $\mu \mathrm{g}$ of cell lysates proteins. Data shown are from one representative experiment with individual points determined in triplicates. Results are presented as the mean $\pm S D$. (b) Sequence of the murine genomic region flanking the transcriptional start sites 1 (TSS1) of the Gdnf mRNA variants expressed in Sertoli cells. Nucleotides are numbered from the TSS1 (marked as arrow). The localization of the regulatory elements is shown as colored boxes. mRNA, messenger RNA; RA, retinoic acid; RARE, RA response elements; RLU, relative luminescence units; $S D$, standard deviation

(Cunningham \& Duester, 2015). We, therefore, tested the hypothesis of the involvement of HDACs in the RA mechanism of action on Gdnf. To this purpose, we tested the effect of two different inhibitors of class I and II HDACs, SAHA, and valproic acid (VPA; Dokmanovic, Clarke, \& Marks, 2007). SAHA treatment significantly reverted the RA-mediated inhibitory effect on Gdnf (Figure 5a) while in the presence of VPA Gdnf basal levels were reduced compared with basal conditions and the inhibitory effect of RA was no longer appreciable (Figure 5b). These suggested that HDACs could be involved in RA-mediated inhibitory regulation (Figure $5 b$ ). To directly verify this hypothesis, we evaluated the acetylation levels of histone $\mathrm{H} 4(\mathrm{H} 4 \mathrm{ac})$ by ChIP-qPCR experiments. Sertoli cells were cultured with or without RA for $2 \mathrm{hr}$, and the enrichment mediated by the anti- $\mathrm{H} 4$ acetylated antibody was measured to evaluate the acetylation status of 105 bp genomic fragment around the TSS1 (Figure 5c). The results demonstrate that RA treatment induces a significant reduction of histone $\mathrm{H} 4$ acetylation levels in this region, suggesting that the RA-mediated negative regulation depends on epigenetic mechanisms.

\section{3 | DISCUSSION}

Previous studies have demonstrated a negative regulation of Gdnf by RA in cultured Sertoli cells as well as in adult testis, but the underlying molecular mechanisms remained unclear (Hasegawa \& Saga,
2012; Sakai et al., 2018). In the present study, we show that the negative regulation of Gdnf by RA also takes place in ex vivo cultured immature testis and staged seminiferous tubules from adult testis. By using in vitro cultures of murine Sertoli cell as an experimental model, we show that Gdnf is directly regulated by RA through a genomic mechanism involving a RARE-DR5 binding site localized on the Gdnf promoter region. The negative regulation requires RAR $\alpha$ and induces a strong decrease of histone $\mathrm{H} 4$ acetylation levels around the TSS1.

The results from ex vivo cultured mouse testis showed that the RA-mediated regulation of Gdnf was detected in the immature but not in the adult testis. However, when staged seminiferous tubules were isolated from adult testis and treated in vitro with RA, Gdnf levels were significantly modulated. We concluded that the concomitant presence of multiple germ cell associations in adult organ cultures can hinder the detection of stage-specific gene regulation in target cells. Overall, our data further support the observation that RA modulates Gdnf levels both in immature and adult mice. The GDNF signaling through the RET/GFRA1 receptor complex is required for postnatal spermatogenesis and is critical for the fate of undifferentiated spermatogonia (Naughton, Jain, Strickland, Gupta, \& Milbrandt, 2006). Modulation of the GDNF levels by RA in immature Sertoli could impact the fate of undifferentiated spermatogonia.

Stra6 is a RA-responsive gene first identified in P19 carcinoma cells, and in adult mice, Stra6 is expressed in Sertoli cells (Bouillet et al, 1997). 
(a)

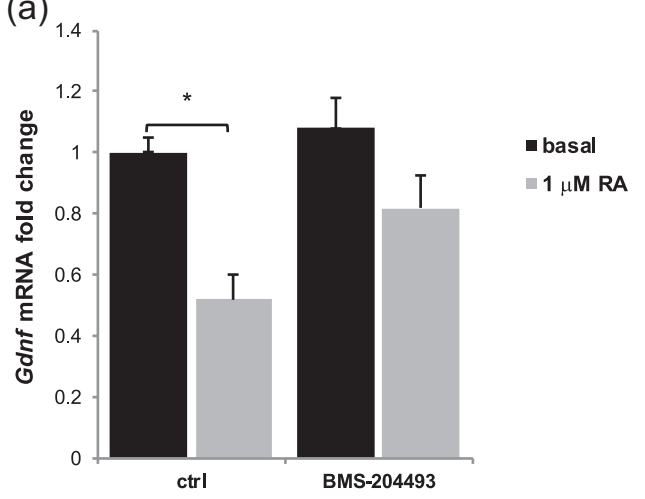

(c)

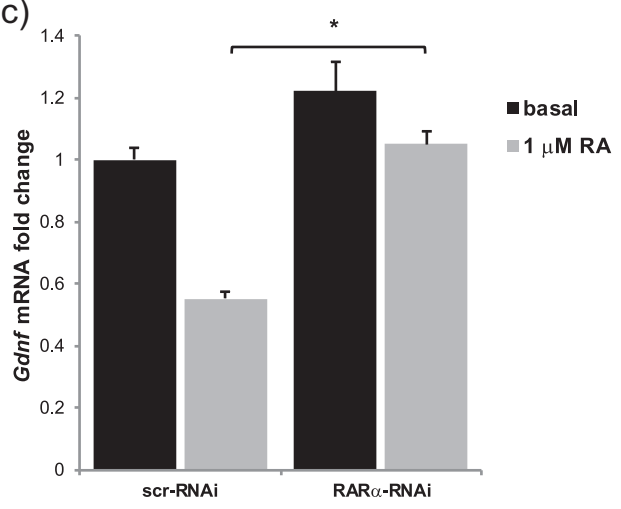

(b)
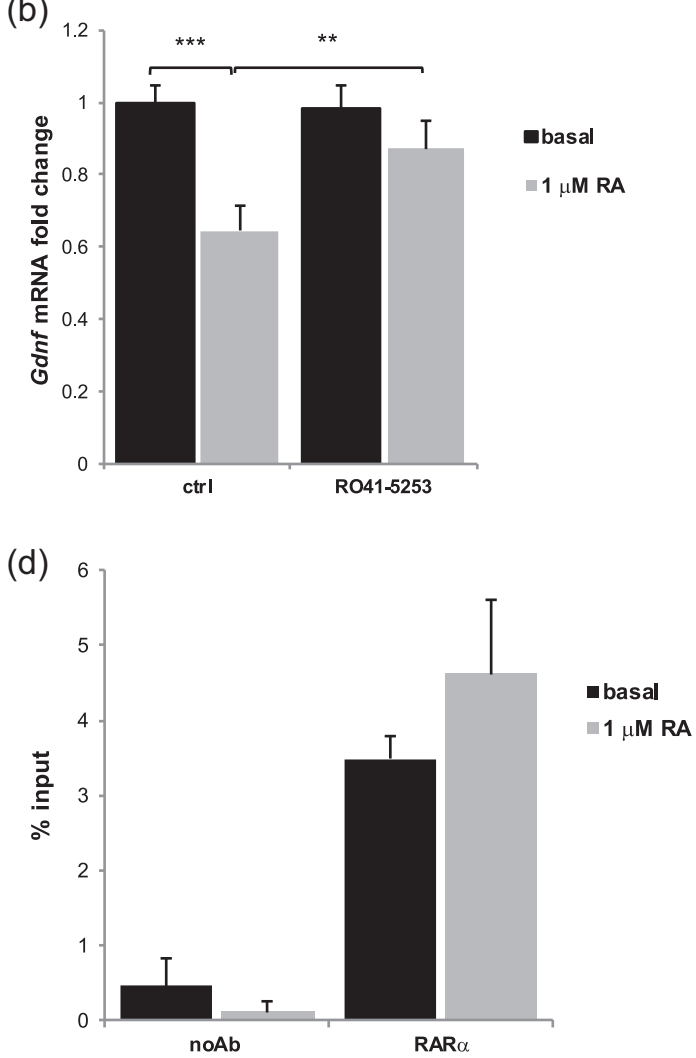

FIGURE 4 RAR $\alpha$ is required for RA-mediated Gdnf downregulation and occupies the RARE-DR5 binding site. (a and b) Sertoli cells were cultured for $24 \mathrm{hr}$ with vehicle or with $10 \mu \mathrm{M}$ BMS-204493 (a) or $3.5 \mu \mathrm{M}$ RO41-5253 (b) in the presence or absence of $1 \mu \mathrm{M}$ RA. Gdnf mRNA levels were measured by real-time PCR, normalized to $\beta$-actin levels and expressed as fold change respect to untreated control. Data are represented as mean \pm SEM from $n=4$ (a) or $n=3$ (b) experiments. (c) Sertoli cells were transfected with scrambled RNAi (scr-RNAi) or with a specific anti-RAR $\alpha$ RNAi (RAR $\alpha$-RNAi) in the presence or absence of $1 \mu \mathrm{M}$ RA for $24 \mathrm{hr}$. Gdnf mRNA levels were quantified by real-time PCR, normalized to $\beta$-actin levels and expressed as fold change respect to untreated control. Data are expressed as mean $\pm S E M$ from $n=2$

experiments and expressed in fold change compared with the untreated control. ${ }^{*} p<.05$. (d) Real-time PCR amplification of the promoter region of Gdnf in the DNA derived from Sertoli cells untreated or treated with $1 \mu \mathrm{M}$ RA for $2 \mathrm{hr}$, immunoprecipitated using the RAR $\alpha$ antibody or with beads without antibody as negative control (noAb). Data shown are from a representative experiment and reported as relative increase to the percentage of input. ${ }^{*} p<.05,{ }^{* *} p<.01,{ }^{* * *} p<.001$. mRNA, messenger RNA; RA, retinoic acid; RAR, RA receptor; RARE, RA response elements; $\mathrm{PCR}$, polymerase chain reaction; SEM, standard error of the mean

In line with previous studies, our analysis showed that the expression profile of Stra6 was stage-specific, mirroring the levels of endogenous RA (Berry et al., 2013; Bouillet et al., 1997; Hasegawa et al., 2013). Interestingly, in staged seminiferous tubules, both Gdnf and Stra6 were modulated by RA treatment only in stages where the endogenous RA levels are low (stages II-VI) and not in stages where RA levels are high (VII-XI). We speculate that from Stage VII onward the target cells are maximally stimulated by endogenous RA and cannot further respond to an exogenous RA trigger.

In immature Sertoli cells, RA induced a time- and dosedependent reduction of Gdnf levels. Protein neosynthesis was found dispensable for RA-mediated Gdnf regulation. In contrast, blocking ongoing transcription for $2 \mathrm{hr}$ lowered Gdnf mRNA by $80 \%$. This suggests that Gdnf transcripts have a short half-life, a feature that may facilitate dynamic control of Gdnf levels in immature Sertoli cells. Interestingly, the analysis of the transcriptome in Sertoli cells points to a plastic control of transcription with most of the transcripts, including Gdnf, dynamically regulated during the first wave of spermatogenesis (Zimmermann et al., 2015).

The murine Gdnf gene has three alternative promoters, among which, promoter 1 is the only active in Sertoli cells. In promoter 1, the TSS1 is located approximately $30 \mathrm{bp}$ downstream from the canonical TATA-box sequence and approximately $900 \mathrm{bp}$ upstream from the putative initiation of translation (Lamberti \& Vicini, 2014). In Sertoli cells transiently transfected with the $1.9 \mathrm{~kb} 5$ ' flanking region of TSS1, RA treatment mediated a significant reduction of luciferase activity measured over the basal condition. This was consistent with the presence of regions implicated in the negative regulation upstream of TSS1 and prompted us to search for putative RARE by in silico analysis. A novel DR5-RARE was identified 307 bp upstream of TSS1. Two lines of evidence suggest that DR5-RARE is involved in RA -modulation of Gdnf expression level: (a) deletion of DR5-RARE in the $1.9 \mathrm{~kb} 5$ ' flanking region of TSS1 reverted the RA-mediate inhibition of luciferase activity in transfected Sertoli cells; (b) DR5-RARE is 
(a)

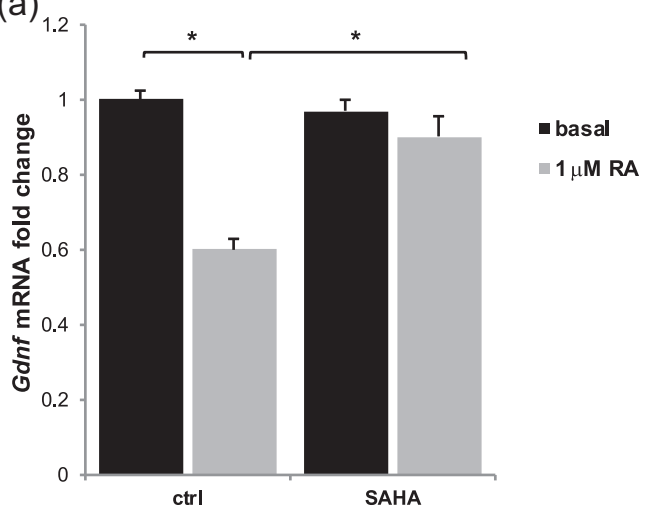

(b)

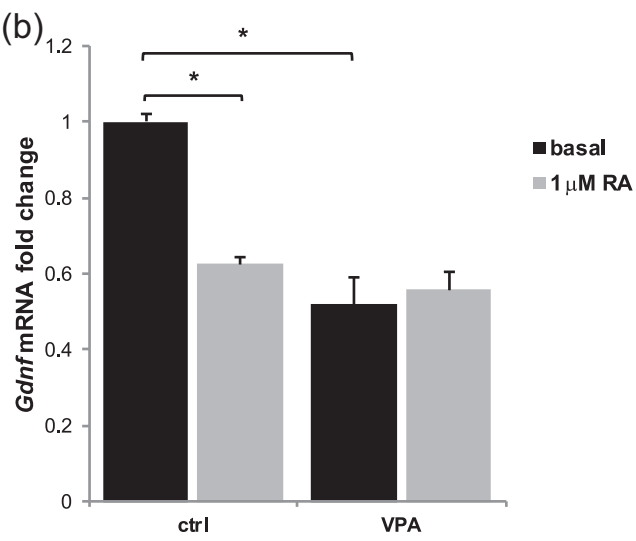

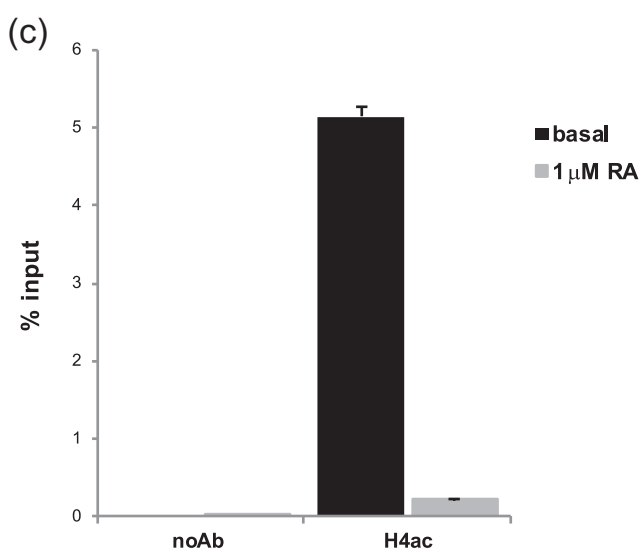

FIGURE 5 Epigenetic mechanisms underly the RA-mediated inhibition of Gdnf. (a and b) Sertoli cells were cultured for $24 \mathrm{hr}$ with vehicle or with $1.25 \mu$ M SAHA (a) or 1 mM VPA (b) in the presence or absence of $1 \mu$ M RA. Gdnf mRNA levels were measured by real-time PCR, normalized to $\beta$-actin levels and expressed as fold change respect to untreated control. Data are represented as mean $\pm S E M$ from $n=3$ experiments. (c) Real-time PCR amplification of the promoter region of Gdnf in the DNA derived from Sertoli cells untreated or treated with $1 \mu \mathrm{M}$ RA for $2 \mathrm{hr}$, immunoprecipitated using the $\mathrm{H} 4$ acetylated antibody or with beads without antibody as negative control (noAb). Data shown are from a representative experiment and reported an increase relative to the percentage of input. ${ }^{*} p<.05$. mRNA, messenger RNA; RA, retinoic acid; PCR, polymerase chain reaction; SEM, standard error of the mean; VPA, valproic acid

occupied by RAR $\alpha$ both in control and RA-treated Sertoli cells as measured by Chip-qPCR analysis. Among the RARs, RAR $\alpha$ is the prominent isoform expressed in Sertoli cells in the adult and prepubertal mice (Vernet et al., 2006), and RAR $\alpha$ is implicated in the specific activation of the RA-dependent signaling in Sertoli cells during the cycle of the seminiferous epithelium (Hasegawa \& Saga, 2012; Vernet et al., 2006). In the present study, we show that ablation of RAR $\alpha$ reverts RA-mediated Gdnf negative regulation, and that in Sertoli cells, Gdnf is a direct target of RA through genomic regulation.

In the classical model of RA-dependent gene regulation, upon RA binding to RARs on RAREs, nuclear receptor coactivators are recruited, which in turn recruit HAT complexes and Trithorax proteins, resulting in chromatin relaxation and gene transcription activation (Cunningham \& Duester, 2015). However, in few documented cases, RA binding to RARs recruits cofactors such as NCOR1, NCOR2, PRC2, and HDAC complexes on the promoter of target genes, leading to gene repression rather than gene activation (reviewed by Cunningham \& Duester, 2015). This mechanism has been described for Hoxb1, Tbx2, and Fgf8
(Kumar, Cunningham, \& Duester, 2016; Kumar \& Duester, 2014; Sakabe, Kokubo, Nakajima, \& Saga, 2012; Studer, Popperl, Marshall, Kuroiwa, \& Krumlauf, 1994). In this study, we show that in cells treated with VPA, Gdnf levels were reduced, whereas SAHA did not modify Gdnf levels, likely due to different specificity towards distinct HDACs as previously reported (Dokmanovic et al., 2007). Moreover, the two compounds may differently impact the proteome and acetylation profile of multiple proteins including key regulators of Gdnf expression (Zhu et al., 2016). Nevertheless, the RA effect on Gdnf expression was reduced in the presence of both HDACs inhibitors.

In line with the model for RA-mediated gene repression proposed by Cunningham and Duester (2015), we show that RA modifies the epigenetic profile of the TSS1 region, inducing deacetylation of histone $\mathrm{H} 4$ compared with the basal condition. Several lines of evidence have previously indicated that DNA methylation and histones modifications impact on Gdnf expression in different cell types (Chen et al., 2006; Uchida et al., 2011; Yu et al., 2014; Zhang et al., 2016). To the best of our knowledge, the present study is the first to 
demonstrate that the expression level of Gdnf in Sertoli cells can be modulated by histone modifications.

To conclude, our findings shed light on a more wide-ranging RA-dependent gene expression regulation and contribute toward a better understanding of the molecular control of the spermatogonial compartment including SSC.

\section{4 | MATERIAL AND METHODS}

\subsection{Testis organ culture}

Adult CD1 mice (3 months) were sacrificed, testes were taken, deprived of tunica albuginea and transferred to a plate with phosphate-buffered saline (PBS; Sigma-Aldrich, Milan, Italy). Testicular fragments of $1 \mathrm{~mm}^{3}$ were cut and placed on steel grids coated with $2 \%$ agar in organ culture dishes containing medium and maintained at $34^{\circ} \mathrm{C}$ in a controlled atmosphere of $5 \% \mathrm{CO}_{2}$. PBS was added to the bottom of the dish to maintain fragments hydration. The RA stock solution was resuspended in dimethyl sulfoxide (DMSO). In RA-treated and control samples, DMSO (Sigma-Aldrich) final concentration did not exceeded $0.1 \%$. At the end of the culture fragments from each point were pulled, washed in PBS and lysed with TRIzol reagent (Invitrogen, Milan, Italy) for the RNA extraction.

\subsection{Isolation and culture of seminiferous tubules}

CD1 mice were sacrificed, testes were taken, deprived of tunica albuginea, and transferred to a plate with PBS. At the stereomicroscope, with 25-50x enlargement, tubules were dissected, and stages of the seminiferous epithelium identified through the different patterns of light absorption by transillumination (Lamberti \& Vicini, 2014). Three groups were isolated: stages IX-XI, stages II-VI, and stages VII-VIII. For each group, $3 \mathrm{~cm}$ total length were measured, collected and cultured at $34^{\circ} \mathrm{C}$ for $4 \mathrm{hr}$ with minimum essential medium (MEM) $1 \times$ (Gibco, Milan, Italy) medium with or without $1 \mu \mathrm{M}$ $\mathrm{RA}$, for subsequent RNA extraction. The RA stock solution was resuspended in DMSO (Sigma-Aldrich). In RA-treated and control samples, DMSO final concentration did not exceeded $0.1 \%$.

\section{3 | Sertoli cell culture and treatments}

Primary Sertoli cell cultures were obtained from 17-day CD1 mice, as previously reported (Lamberti \& Vicini, 2014). Briefly, isolated testes were washed twice with Hank's solution (SigmaAldrich) and the tunica albuginea was removed. Subsequently, testes were chopped, transferred to Hank's solution containing 0.002\% DNAse I (Roche, Milan, Italy), 0.25\% Trypsin (Difco, Milan, Italy) and agitated at $32^{\circ} \mathrm{C}$ for $13 \mathrm{~min}$. The digestion was blocked by adding $10 \%$ fetal bovine serum. The seminiferous tubules were deposited for $4 \mathrm{~min}$ at unit gravity and washed twice with Hank's solution. The second digestion was performed with a solution of Hank's, $0.002 \%$ DNAse I and $0.2 \%$ of Collagenase $\mathrm{A}$ (Roche) at $32^{\circ} \mathrm{C}$ for $20 \mathrm{~min}$, with agitation. In the end, the fragmented tubules were deposited at unit gravity, washed with Hank's solution, collected in a polystyrene tube and subjected to centrifugation at $18 \mathrm{~g}$ for $2 \mathrm{~min}$. The pellet was then resuspended in MEM $1 \times$, containing $20 \mathrm{mM}$ glutamine, Hepes $0.01 \mathrm{M}$ (non-essential amino acids $0.1 \mathrm{mM}$ and $1 \%$ antibiotics). Cells were maintained at $34^{\circ} \mathrm{C}$ in a controlled atmosphere of $5 \%$ $\mathrm{CO}_{2}$. With this procedure, a monolayer is formed consisting exclusively of Sertoli cells and germ cells. The residual germ cells were eliminated at $48 \mathrm{hr}$ of culture with a short hypotonic treatment $(2.5 \mathrm{~min}$ at room temperature with $20 \mathrm{mM}$ Tris- $\mathrm{HCl}$; Galdieri \& Zani, 1981). The purity of Sertoli cell cultures was assessed by morphological analysis and by immunolocalization of smooth muscle actin (a-SMA), a marker of peritubular myoid cells. The purity of Sertoli cells was routinely higher than $95 \%$. Sertoli cell cultures were used after $24 \mathrm{hr}$ from hypotonic treatment. Sertoli cells were treated with: different concentration of RA (alltrans-RA; Sigma-Aldrich); ActD (5 $\mathrm{gg} / \mathrm{ml}$; Sigma-Aldrich); CHX $(10 \mu \mathrm{g} / \mathrm{ml}$; Sigma-Aldrich); BMS-204493 (10 $\mu \mathrm{M}$; Sigma-Aldrich); RO41-5253 (3.5 $\mu \mathrm{M}$; Santa Cruz Biotechnology); VPA (1 mM; Sigma-Aldrich); SAHA (1.25 $\mu \mathrm{M}$; Sigma-Aldrich).

\subsection{RNA extraction and real-time PCR}

Total RNA extraction was performed using the TRIzol reagent (Invitrogen) following the manufacturer's instructions. The obtained RNA was quantified by Nanodrop 1000 (Thermo Fisher Scientific, Milan, Italy). RNA quality was assessed by agarose gel electrophoresis and by calculating the ratio of the absorbance at 260 and $280 \mathrm{~nm}$ (A260/280) that was routinely comprised between 1.9 and 2. The complementary DNA (cDNA) synthesis, from $1 \mu \mathrm{g}$ of RNA for each sample, was performed using the High-Capacity RNA-tocDNA $^{\text {TM }}$ Kit (Thermo Fisher Scientific, Waltham, MA) following the manufacturer's instructions. In the control samples, reverse transcriptase was omitted to monitor genomic DNA contamination. The list of genes analyzed, and the respective primers used are reported in Table S1. Primer pairs were designed using PrimerBLAST (https://www.ncbi.nlm.nih.gov/tools/primer-blast/) and nucleotide sequences available at NCBI databases. The cDNA was subjected to Real-Time PCR analysis with FluoCycleTMII SYBR Green Mix (Euroclone) in 7500 Real-Time PCR System (Applied Biosystems, Life Technologies, Monza, Italy). Blank controls were assayed in each reaction and for each primer pair to detect reagent contamination. Data were analyzed with the comparative $2^{-\Delta \Delta G}$ method using $\beta$-actin as reference gene and control conditions as calibrator (Schmittgen \& Livak, 2008). Each real-time PCR assay was repeated at least two times, using duplicate samples in three different experiments. The mean values with standard error of the mean (SEM) were used for comparison. 


\section{5 | siRNA}

To interfere with RAR $\alpha$ expression, primary Sertoli cells were transfected with scrambled siRNA (MISSION siRNA Universal Negative control; Sigma-Aldrich) or with siRNA specific for RAR $\alpha$ (Invitrogen; sequences in Table S1; Lamberti \& Vicini, 2014). After the hypotonic treatment, Sertoli cells were cultured in the absence of antibiotics and transfected for $5 \mathrm{hr}$ with $5 \mu \mathrm{l} /$ well Lipofectamine 2000 (Invitrogen) and $250 \mathrm{pmol}$ of the anti-RAR $\alpha$ siRNA or not specific siRNA in Opti-MEM medium (Invitrogen). At the end of the treatment, the medium was removed and MEM 1×, containing $20 \mathrm{mM}$ glutamine, Hepes $0.01 \mathrm{M}$ (non-essential $0.1 \mathrm{mM}$ amino acids and antibiotics) was added for the following $24 \mathrm{hr}$ in the presence or absence of $1 \mu \mathrm{M}$ RA. Gdnf expression level was evaluated by realtime PCR and CDNA levels were standardized by normalizing to a $\beta$-actin control. The expression levels are presented as fold induction over the basal condition of the not specific siRNA sample. The results are from two independent experiments and are expressed as the mean \pm SEM.

\section{6 | RARE-DR5 identification and mutagenesis}

The putative RARE-DR5 (5'-tGATCAgGgAGAGGTCA-3') binding site on murine Gdnf promoter was identified using the Softberry NSITE Program (Version 5.2013, http://www.softberry.com). To obtain internal deletions of putative RARE-DR5 on the Gdnf promoter, a plasmid containing the $-1,857 /+109$ genomic fragment (+1 position corresponds to TSS1; Lamberti \& Vicini, 2014) was modified using a PCR associated technique (QuikChange II XL Site-Directed Mutagenesis Kit; Agilent Technologies, Milano, Italy) to obtain $-1,857 /+109-\Delta$ (RARE-DR5)-Pgl2 plasmid. Forward and reverse primers, carrying the appropriate deletion of the region of interest were designed to keep the mutation in the middle of the primer. After PCR extension, 2-5 $\mu$ l of the reaction was used to transform XL -10 gold bacteria (Stratagene, Milano, Italy). Several bacterial colonies were selected and sequenced to evaluate the introduction of the desired mutation. The sequence of primers used for mutagenesis are listed in Table S1.

\section{7 | Sertoli cell transient transfection and luciferase activity assay}

Sertoli cell transfection was performed as described, with minor modifications (Vicini \& Conti, 1997). Briefly, cells were deprived of the antimicrobial solution for at least $24 \mathrm{hr}$ and then transfected with Lipofectamine 2000 (Invitrogen, Monza, Italy) and $1.5 \mu \mathrm{g} / \mathrm{ml}$ of promoterless construct (control-Pg|2), or $-1,857 /+109-\mathrm{Pg} \mid 2$, or $-1,857 /+109-\Delta$ (RARE-DR5)-Pgl2. After $5 \mathrm{hr}$, the medium was removed, and the cells cultured with MEM $1 \times$ (Gibco) with or without $1 \mu \mathrm{M}$ RA for the following $24 \mathrm{hr}$. Cells were washed and lysed using $1 \times$ Passive lysis buffer (Promega, Milan, Italy), according to the manufacturer's directions. The cell lysates were centrifuged $16,000 \mathrm{~g}$ for $2 \mathrm{~min}$ at $4^{\circ} \mathrm{C}$, and aliquots of the supernatants were assayed for luciferase activity and for protein content (BCA assay; Thermo Fisher Scientific, Milan, Italy). For each sample, luciferase activity was tested in duplicate, mixing $20 \mu \mathrm{l}$ cell extract with $100 \mu \mathrm{l}$ Luciferase Assay Reagent (Promega) and the light produced was measured in GloMax Discover luminometer (Promega). The activity was expressed in relative luminescence units and normalized for $\mu \mathrm{g}$ of proteins. Each construct was assayed in duplicate in at least three different experiments. Data were expressed as mean \pm standard deviation (SD).

\subsection{ChiP assays}

ChiP assay was performed as described, with minor modifications (Iosue et al., 2013). Sertoli cell cultures were treated for $2 \mathrm{hr}$ in with or without $1 \mu \mathrm{M}$ RA, then cross-linked with $1 \%$ formaldehyde (SigmaAldrich). After two lysis steps, the cell chromatin was sonicated to obtain fragments of about $800-500 \mathrm{bp}$. The size of the fragments was evaluated by electrophoretic run on $1 \%$ agarose gel. Subsequently the chromatin was diluted in a chromatin dilution buffer (0.01\% sodium dodecyl sulfate [SDS], 1.1\% Triton X-100, $1.2 \mathrm{mM}$ ethylenediaminetetraacetic acid [EDTA], $16.7 \mathrm{mM}$ Tris- $\mathrm{HCl}, \mathrm{pH} 8.1$, $167 \mathrm{mM} \mathrm{NaCl}$ ). Sonicated chromatin was immunoprecipitated overnight at $4^{\circ} \mathrm{C}$ with anti-H4-Ac (5 $\mathrm{g}$, 06-866, Millipore) or anti-RAR $\alpha$ (8 $\mu \mathrm{g}$, sc-551; Santa Cruz Biotechnology) linked to Dynabeads Protein G (Thermo Fisher Scientific, Milan, Italy) or with Dynabeads alone as negative control. The immunoprecipitated material linked to the beads was then washed four times with low salt concentration buffer (0.1\% SDS, 1\% Triton X-100, 2 mM EDTA, 20 mM Tris- $\mathrm{HCl}$, pH 8.1, $150 \mathrm{mM} \mathrm{NaCl}), 3$ times with high salt concentration buffer $(0.1 \%$ SDS, 1\% Triton X-100, 2 mM EDTA, 20 mM Tris- $\mathrm{HCl}$, pH 8.1, 500 mM $\mathrm{NaCl}$ ), once with Tris/EDTA buffer and finally eluted with elution buffer ( $1 \% \mathrm{SDS}, 0.1 \mathrm{M} \mathrm{NaHCO})_{3}$ for $30 \mathrm{~min}$ at $37^{\circ} \mathrm{C}$. DNA was reverse cross-linked with $0.2 \mathrm{M} \mathrm{NaCl}$ and recovered with the phenol/chloroform extraction followed by ethanol precipitation. Immunoprecipitated DNA was amplified by real-time PCR primers targeting $105 \mathrm{bp}$ including the TSS1 or with primers targeting $142 \mathrm{bp}$ including DR5-RARE on the Gdnf promoter. Primers targeting the intron 1 of Gdnf, were used as negative controls. Real-time PCR was performed as detailed above. Each experiment was repeated two times with similar results. The sequences of primers used for ChiP assay are shown in Table S1. Data were analyzed by the percentage of input method (http://www.thermofisher.com/it/en/home/lifescience/epigenetics-noncoding-rna-research/chromatin-remodeling/ chromatin-immunoprecipitation-chip/chip-analysis.html).

\section{9 | Statistical analysis}

Statistical analysis was performed using the Sigma Plot 11.0 program. Quantitative data are shown as the mean $\pm S E M$, mean $\pm S D$, or percentile plots for data obtained using human material. To define the 
significance of the differences between two groups, data were analyzed using a $t$ test. To compare many groups, data were analyzed using a one-way analysis of variance followed by a post hoc Dunn's method. The significance level was fixed at $\alpha=.05$.

\section{ACKNOWLEDGMENT}

This study was supported by grants from Sapienza University (Ateneo 2018).

\section{CONFLICT OF INTERESTS}

The authors declare that there are no conflict of interests.

\section{ORCID}

Elena Vicini (D) http://orcid.org/0000-0003-0399-0145

\section{REFERENCES}

Balmer, J. E., \& Blomhoff, R. (2005). A robust characterization of retinoic acid response elements based on a comparison of sites in three species. The Journal of Steroid Biochemistry and Molecular Biology, 96, 347-354.

Berry, D. C., Jacobs, H., Marwarha, G., Gely-Pernot, A., O'Byrne, S. M., DeSantis, D., ... Ghyselinck, N. B. (2013). The STRA6 receptor is essential for retinol-binding protein-induced insulin resistance but not for maintaining vitamin A homeostasis in tissues other than the eye. The Journal of Biological Chemistry, 288, 24528-24539.

Bhang, D. H., Kim, B. J., Kim, B. G., Schadler, K., Baek, K. H., Kim, Y. H., .. Ryeom, S. (2018). Testicular endothelial cells are a critical population in the germline stem cell niche. Nature Communications, 9, 4379.

Boitani, C., Di Persio, S., Esposito, V., \& Vicini, E. (2016). Spermatogonial cells: Mouse, monkey and man comparison. Seminars in Cell and Developmental Biology, 59, 79-88.

Bouillet, P., Sapin, V., Chazaud, C., Messaddeq, N., Decimo, D., Dolle, P., \& Chambon, P. (1997). Developmental expression pattern of Stra6, a retinoic acid-responsive gene encoding a new type of membrane protein. Mechanisms of Development, 63, 173-186.

Chen, L. Y., Willis, W. D., \& Eddy, E. M. (2016). Targeting the Gdnf Gene in peritubular myoid cells disrupts undifferentiated spermatogonial cell development. Proceedings of theNational AcademyofSciences of the United States of America, 113, 1829-1834.

Chen, P. S., Peng, G. S., Li, G., Yang, S., Wu, X., Wang, C. C., ... Hong, J. S. (2006). Valproate protects dopaminergic neurons in midbrain neuron/ glia cultures by stimulating the release of neurotrophic factors from astrocytes. Molecular Psychiatry, 11, 1116-1125.

Cunningham, T. J., \& Duester, G. (2015). Mechanisms of retinoic acid signalling and its roles in organ and limb development. Nature Reviews. Molecular Cell Biology, 16, 110-123.

Dokmanovic, M., Clarke, C., \& Marks, P. A. (2007). Histone deacetylase inhibitors: Overview and perspectives. Molecular Cancer Research, 5, 981-989.

Dovere, L., Fera, S., Grasso, M., Lamberti, D., Gargioli, C., Muciaccia, B., ... Vicini, E. (2013). The niche-derived glial cell line-derived neurotrophic factor (GDNF) induces migration of mouse spermatogonial stem/ progenitor cells. PLoS One, 8:e59431.

Endo, T., Romer, K. A., Anderson, E. L., Baltus, A. E., de Rooij, D. G., \& Page, D. C. (2015). Periodic retinoic acid-STRA8 signaling intersects with periodic germ-cell competencies to regulate spermatogenesis. Proceedings of the National Academy of Sciences of the United States of America, 112, E2347-E2356.

Ertesvag, A., Aasheim, H. C., Naderi, S., \& Blomhoff, H. K. (2007). Vitamin A potentiates CpG-mediated memory B-cell proliferation and differentiation: Involvement of early activation of p38MAPK. Blood, 109, 3865-3872.
Galdieri, M., \& Zani, B. (1981). Hormonal induced changes in sertoli cell glycoproteins. Cell Biology International Reports, 5, 111.

Golden, J. P., DeMaro, J. A., Osborne, P. A., Milbrandt, J., \& Johnson, E. M., Jr. (1999). Expression of neurturin, GDNF, and GDNF family-receptor mRNA in the developing and mature mouse. Experimental Neurology, 158, 504-528.

Grasso, M., Fuso, A., Dovere, L., de Rooij, D. G., Stefanini, M., Boitani, C., \& Vicini, E. (2012). Distribution of GFRA1-expressing spermatogonia in adult mouse testis. Reproduction, 143, 325-332.

Griswold, M. D. (2016). Spermatogenesis: The commitment to meiosis. Physiological Reviews, 96, 1-17.

Hasegawa, K., Namekawa, S. H., \& Saga, Y. (2013). MEK/ERK signaling directly and indirectly contributes to the cyclical self-renewal of spermatogonial stem cells. Stem Cells, 31, 2517-2527.

Hasegawa, K., \& Saga, Y. (2012). Retinoic acid signaling in Sertoli cells regulates organization of the blood-testis barrier through cyclical changes in gene expression. Development, 139, 4347-4355.

Hogarth, C. A., Arnold, S., Kent, T., Mitchell, D., Isoherranen, N., \& Griswold, M. D. (2015). Processive pulses of retinoic acid propel asynchronous and continuous murine sperm production. Biology of Reproduction, 92, 37.

Iosue, I., Quaranta, R., Masciarelli, S., Fontemaggi, G., Batassa, E. M., Bertolami, C., ... Fazi, F. (2013). Argonaute 2 sustains the gene expression program driving human monocytic differentiation of acute myeloid leukemia cells. Cell Death and Disease, 4:e926.

Johnston, D. S., Olivas, E., DiCandeloro, P., \& Wright, W. W. (2011). Stagespecific changes in GDNF expression by rat Sertoli cells: A possible regulator of the replication and differentiation of stem spermatogonia. Biology of Reproduction, 85, 763-769.

Kanatsu-Shinohara, M., Ogonuki, N., Inoue, K., Miki, H., Ogura, A., Toyokuni, S., \& Shinohara, T. (2003). Long-term proliferation in culture and germline transmission of mouse male germline stem cells. Biology of Reproduction, 69, 612-616.

Kumar, S., Cunningham, T. J., \& Duester, G. (2016). Nuclear receptor corepressors Ncor1 and Ncor2 (Smrt) are required for retinoic aciddependent repression of Fgf8 during somitogenesis. Developmental Biology, 418, 204-215.

Kumar, S., \& Duester, G. (2014). Retinoic acid controls body axis extension by directly repressing Fgf8 transcription. Development, 141, 2972-2977.

Lamberti, D., \& Vicini, E. (2014). Promoter analysis of the gene encoding GDNF in murine Sertoli cells. Molecular and Cellular Endocrinology, 394, 105-114.

Meng, X., de Rooij, D. G., Westerdahl, K., Saarma, M., \& Sariola, H. (2001). Promotion of seminomatous tumors by targeted overexpression of glial cell line-derived neurotrophic factor in mouse testis. Cancer Research, 61, 3267-3271.

Meng, X., Lindahl, M., Hyvonen, M. E., Parvinen, M., de Rooij, D. G., Hess, M. W., ... Sariola, H. (2000). Regulation of cell fate decision of undifferentiated spermatogonia by GDNF. Science, 287, 1489-1493.

Morales, C., \& Griswold, M. D. (1987). Retinol-induced stage synchronization in seminiferous tubules of the rat. Endocrinology, 121, 432-434.

Naughton, C. K., Jain, S., Strickland, A. M., Gupta, A., \& Milbrandt, J. (2006). Glial cell-line derived neurotrophic factor-mediated RET signaling regulates spermatogonial stem cell fate. Biology of Reproduction, 74, 314-321.

Raverdeau, M., Gely-Pernot, A., Feret, B., Dennefeld, C., Benoit, G., Davidson, I., ... Ghyselinck, N. B. (2012). Retinoic acid induces Sertoli cell paracrine signals for spermatogonia differentiation but cell autonomously drives spermatocyte meiosis. Proceedings of the National Academy of Sciences of the United States of America, 109, 16582-16587.

Sakabe, M., Kokubo, H., Nakajima, Y., \& Saga, Y. (2012). Ectopic retinoic acid signaling affects outflow tract cushion development through 
suppression of the myocardial Tbx2-Tgfbeta2 pathway. Development, 139, 385-395.

Sakai, M., Masaki, K., Aiba, S., Tone, M., \& Takashima, S. (2018). Expression dynamics of self-renewal factors for spermatogonial stem cells in the mouse testis. Journal of Reproduction and Development, 64, 267-275.

Schmittgen, T. D., \& Livak, K. J. (2008). Analyzing real-time PCR data by the comparative C(T) method. Nature Protocols, 3, 1101-1108.

Sharma, M., \& Braun, R. E. (2018). Cyclical expression of GDNF is required for spermatogonial stem cell homeostasis. Development, 145, pii: $\operatorname{dev} 151555$.

Studer, M., Popperl, H., Marshall, H., Kuroiwa, A., \& Krumlauf, R. (1994). Role of a conserved retinoic acid response element in rhombomere restriction of Hoxb-1. Science, 265, 1728-1732.

Tadokoro, Y., Yomogida, K., Ohta, H., Tohda, A., \& Nishimune, Y. (2002). Homeostatic regulation of germinal stem cell proliferation by the GDNF/FSH pathway. Mechanisms of Development, 113, 29-39.

Uchida, A., Kishi, K., Aiyama, Y., Miura, K., Takase, H. M., Suzuki, H., ... Kanai, Y. (2016). In vivo dynamics of GFRalpha1-positive spermatogonia stimulated by GDNF signals using a bead transplantation assay. Biochemical and Biophysical Research Communications, 476, 546-552.

Uchida, S., Hara, K., Kobayashi, A., Otsuki, K., Yamagata, H., Hobara, T., ... Watanabe, Y. (2011). Epigenetic status of Gdnf in the ventral striatum determines susceptibility and adaptation to daily stressful events. Neuron, 69, 359-372.

van Pelt, A. M., \& de Rooij, D. G. (1990a). Synchronization of the seminiferous epithelium after vitamin $A$ replacement in vitamin A-deficient mice. Biology of Reproduction, 43, 363-367.

van Pelt, A. M., \& de Rooij, D. G. (1990b). The origin of the synchronization of the seminiferous epithelium in vitamin A-deficient rats after vitamin A replacement. Biology of Reproduction, 42, 677-682.

van Pelt, A. M., \& de Rooij, D. G. (1991). Retinoic acid is able to reinitiate spermatogenesis in vitamin A-deficient rats and high replicate doses support the full development of spermatogenic cells. Endocrinology, 128, 697-704.

Vernet, N., Dennefeld, C., Rochette-Egly, C., Oulad-Abdelghani, M., Chambon, P., Ghyselinck, N. B., \& Mark, M. (2006). Retinoic acid metabolism and signaling pathways in the adult and developing mouse testis. Endocrinology, 147, 96-110.
Vicini, E., \& Conti, M. (1997). Characterization of an intronic promoter of a cyclic adenosine 3',5'-monophosphate (cAMP)-specific phosphodiesterase gene that confers hormone and cAMP inducibility. Molecular Endocrinology, 11, 839-850.

Yomogida, K., Yagura, Y., Tadokoro, Y., \& Nishimune, Y. (2003). Dramatic expansion of germinal stem cells by ectopically expressed human glial cell line-derived neurotrophic factor in mouse Sertoli cells. Biology of Reproduction, 69, 1303-1307.

Yu, Z. Q., Zhang, B. L., Ni, H. B., Liu, Z. H., Wang, J. C., Ren, Q. X., ... Gao, D. S. (2014). Hyperacetylation of histone H3K9 involved in the promotion of abnormally high transcription of the gdnf gene in glioma cells. Molecular Neurobiology, 50, 914-922.

Zhang, B. L., Liu, J., Lei, Y., Xiong, Y., Li, H., Lin, X., ... Gao, D. S. (2016). An epigenetic mechanism of high Gdnf transcription in glioma cells revealed by specific sequence methylation. Molecular Neurobiology, 53 , 4352-4362.

Zhu, X., Liu, X., Cheng, Z., Zhu, J., Xu, L., Wang, F., ... Wu, Q. (2016). Quantitative analysis of global proteome and lysine acetylome reveal the differential impacts of VPA and SAHA on HL60 cells. Scientific Reports, 6, 19926.

Zimmermann, C., Stevant, I., Borel, C., Conne, B., Pitetti, J. L., Calvel, P., ... Nef, S. (2015). Research resource: The dynamic transcriptional profile of sertoli cells during the progression of spermatogenesis. Molecular Endocrinology, 29, 627-642.

\section{SUPPORTING INFORMATION}

Additional supporting information may be found online in the Supporting Information section.

How to cite this article: Saracino R, Capponi C, Di Persio S, et al. Regulation of Gdnf expression by retinoic acid in Sertoli cells. Mol Reprod Dev. 2020;1-11.

https://doi.org/10.1002/mrd.23323 